\title{
ABSTRACT MARTINGALES IN BANACH SPACES
}

\author{
J. J. UHL, JR.
}

ABSTRACT. The concept of martingale is generalized from probability theory to the setting of Banach spaces. Convergent martingales are characterized. An application to a Radon-Nikodym theorem for vector measures is given.

1. Abstract martingales. Let $X$ be a Banach space and $\left\{E_{\tau}, \tau \in I\right\}$ be a uniformly bounded net of continuous linear projections of $X$ into itself satisfying $E_{\tau} E_{\tau_{1}}=E_{\tau_{1}} E_{\tau}=E_{\tau_{1}}$ for $\tau \geqq \tau_{1} \in I$. A net $\left\{x_{\tau}\right.$, $\tau \in I\} \subset X$ indexed by the same directed set $I$ will be called an abstract martingale and denoted by $\left\{x_{\tau}, E_{\tau}, \tau \in I\right\}$ if $E_{\tau_{1}}\left(x_{\tau_{2}}\right)=X_{\tau_{1}}$ for $\tau_{1}, \tau_{2} \in I, \tau_{1} \leqq \tau_{2}$. Clearly abstract martingales are generalizations of the martingales of probability theory [2], [5], and [8]. On the other hand there are many examples of abstract martingales which do not arise as martingales in the sense of probability theory (see [3, pp. 426$427]$ ). The purpose of this note is to characterize strongly convergent abstract martingales and to indicate briefly some applications including a new Radon-Nikodym theorem for vector valued measures.

THEOREM 1. Let $\left\{x_{\tau}, E_{\tau}, \tau \in I\right\}$ be an abstract martingale in a Banach space $X$. Then $\left\{x_{\tau}, E_{\tau}, \tau \in I\right\}$ is strongly convergent (i.e. $\lim _{\tau} x_{\tau}$ exists strongly in $X)$ if and only if there exists a weakly compact set $K \subset X$ such that for each $\epsilon>0$ there exists a $\tau_{\epsilon} \in I$ such that $\tau \in I, \tau \geqq \tau_{\text {e }}$ implies $x_{r} \in K+\epsilon U(=\{k+\epsilon u: k \in K, u \in U\})$ where $U$ is the open unit ball of $X$.

Proof. The necessity is immediate: let $K=\left\{\lim _{\tau} x_{\tau}\right\}$. Then $\left\{x_{\tau}, \tau \in I\right\}$ is eventually in $K+\epsilon U$ for every choice of $\epsilon$. To prove the sufficiency of the condition, let $K$ be as in the hypothesis and select $\left\{\tau_{n}\right\} \subset I$ by choosing $\tau_{1}$ such that $\tau \geqq \tau_{1}$ implies $x_{\tau} \in K+U$ and $\tau_{n} \geqq \tau_{n-1}$ such that $x_{\tau} \in K+(1 / n) U$ for $\tau \geqq \tau_{n}$. Now for each $\tau \in I$, choose $z_{\tau}$ according to the following criteria:

(i) if $\tau \geqq \tau_{n}$ for all $n$, then $x_{\tau} \in K$ and $z_{\tau}$ is taken to be $x_{\tau}$;

(ii) if $\tau \geqq \tau_{n_{0}}$ and it is not the case that $\tau \geqq \tau_{n_{0}+1}$, choose $z_{\tau} \in K$ such that $\left\|z_{\tau}-x_{\tau}\right\|<1 / n_{0}$;

(iii) if there exists no $n$ such that $\tau \geqq \tau_{n}$, choose $z_{\tau} \in K$ arbitrarily.

Received by the editors April 6, 1970.

AMS 1969 subject classifications. Primary 4610; Secondary 2850, 6040.

Key words and phrases. Martingale, Banach spaces, projection, vector measures, Radon-Nikodym. 
Now consider the net $\left\{z_{r}, \tau \in I\right\} \subset K$. Since $K$ is weakly compact, there exists a subnet $\left\{z_{\alpha}, \alpha \in A\right\}$ of $\left\{z_{r}, \tau \in I\right\}$ converging weakly to some point $x \in K$. Now let $f: A \rightarrow I$ be a function which guarantees that $\left\{z_{\alpha}, \alpha \in A\right\}$ is a subnet of $\left\{z_{\tau}, \tau \in I\right\}$ [6, p. 70] and define $\left\{x_{\alpha}, \alpha \in A\right\}$ by $x_{\alpha}=x_{f(\alpha)}$. Then $\left\{x_{\alpha}, \alpha \in A\right\}$ is a subnet of $\left\{x_{\tau}, \tau \in I\right\}$ and $\left\|x_{\alpha}-z_{\alpha}\right\|=\left\|x_{f(\alpha)}-z_{f(\alpha)}\right\|$. Moreover if $x^{*} \in X^{*}$, the space of bounded linear functionals on $X$, one has

$$
\begin{aligned}
\lim _{\alpha}\left|x^{*}\left(x_{\alpha}-x\right)\right| & \leqq \lim _{\alpha}\left|x^{*}\left(x_{\alpha}-z_{\alpha}\right)\right|+\lim _{\alpha}\left|x^{*}\left(z_{\alpha}-x\right)\right| \\
& \leqq \| x^{*}|| \lim _{n} \frac{1}{n}+0=0 .
\end{aligned}
$$

Hence $\lim _{\alpha} x_{\alpha}=x$ weakly in $X$. Also since $\left\{x_{\alpha}, \alpha \in A\right\}$ is a subnet of $\left\{x_{\tau}, \tau \in I\right\}, x_{\tau}=\lim _{\alpha} E_{\tau}\left(x_{\alpha}\right)$ strongly for all $\tau \in I$. Accordingly if $\tau \in I$ and $x^{*} \in X^{*}$,

$$
x^{*}\left(x_{\tau}-E_{\tau}(x)\right)=x^{*}\left(E_{\tau}\left(x_{\tau}-x\right)\right)=\lim _{\alpha} x^{*} E_{\tau}\left(x_{\alpha}-x_{\alpha}\right)=0,
$$

since $\lim _{\alpha} x_{\alpha}=x$ weakly and $x_{\tau}=\lim _{\alpha} E_{\tau}\left(x_{\alpha}\right)$ strongly. Hence $x_{\tau}$ $=E_{\tau}(x)$ for all $\tau \in I$.

Finally it will be shown that $\lim _{\tau} x_{\tau}=x$ strongly in $X$. Let $M=\left\{z \in X: E_{\tau}(z)=z\right.$ for some $\left.\tau \in I\right\}$. The facts that $I$ is directed and that $E_{\tau} E_{\tau_{1}}=E_{\tau_{1}} E_{\tau}=E_{\tau_{1}}$ for $\tau \geqq \tau_{1}$ ensure that $M$ is a linear manifold in $X$. But, since $\lim _{\alpha} x_{\alpha}=x$ weakly and $\left\{x_{\alpha}\right\} \subset M, x \in$ weak closure of $M$ and therefore to the strong closure of the linear manifold $M$. Now let $P=\sup _{\tau}\left\|E_{\tau}\right\|$ and $\epsilon>0$ be given. Choose $y \in M$ such that $\|x-y\|<\epsilon / P+1$. Selecting $\tau_{0} \in T$ such that $E_{\tau_{0}}(y)=y$, one finds that for $\tau \geqq \tau_{0}, \quad E_{\tau}(y)=y$ since $E_{\tau}(y)=E_{\tau} E_{\tau_{0}}(y)=E_{\tau_{0}}(y)=y$. Hence for $\tau \geqq \tau_{0}$,

$$
\begin{aligned}
\left\|x_{\tau}-x\right\| & =\left\|E_{\tau}(x)-x\right\| \leqq\left\|E_{\tau}(x)-y\right\|+\|y-x\| \\
& =\left\|E_{\tau}(x-y)\right\|+\|y-x\|<P_{\epsilon} /(P+1)+\epsilon /(P+1)=\epsilon .
\end{aligned}
$$

A considerable shortening of the proof of Theorem 1 results in

Corollary 2. An abstract martingale is strongly convergent if and only if it is weakly convergent.

Also immediate is

COROLlaRy 3. An abstract martingale in a reflexive Banach space is convergent if and only if it is bounded. 
2. Applications to martingales and integral representation of vector measures. If $X$ is a reflexive Banach space, and $(\Omega, \Sigma, \mu)$ is a finite measure space, Scalora and Chatterji have shown that a martingale $\left\{f_{n}, B_{n}\right\}$ in $L^{p}(\Omega, \Sigma, \mu, X)\left(=L^{p}(X)\right)$ converges for $1<p<\infty$ if and only if $\left\{f_{n}, B_{n}\right\}$ is bounded [2, Theorem 3]. Since the spaces $L^{p}(X)(1<p<\infty)$ are reflexive, for reflexive $X$, Corollary 3 contains this result as a special case. In the case $p=1$, Chatterji and Scalora prove that a martingale $\left\{f_{n}, B_{n}\right\}$ in $L^{1}(X)$ is convergent. if it is bounded and uniformly integrable for reflexive Banach spaces $X$. But, as Chatterji points out [2, p. 145], this assumption guarantees that $\left\{f_{n}, B_{n}\right\}$ lies in a weakly compact subset of $L^{1}(X)$. Thus Theorem 1 and its corollary contain the full Chatterji-Scalora theorem on mean convergence of martingales in $L^{p}(X)(1 \leqq p<\infty)$. Of course this theorem gives no direct information on almost sure convergence of martingales. On the other hand such information is not to be expected from a theorem of the nature of Theorem 1 .

The connection between martingales and derivatives of set functions is well known[8]. The final considerations of this note are devoted to that subject.

Let $(\Omega, \Sigma, \mu)$ be a finite measure space. A partition $\pi=\left\{E_{n}\right\}$ is a finite disjoint collection of sets in $\Sigma$ such that $U_{n} E_{n}=\Omega$. The collection of partitions $P$ becomes a directed set if one defines $\pi_{1} \leqq \pi_{2}$ if $E \in \pi_{1}$ implies $E$ is a union of members of $\pi_{2}$. Now let $F$ be a $\mu$-continuous countably additive set function defined on $\Sigma$ with values in a Banach space $X$. Define for each partition $\pi=\left\{E_{n}\right\}$ the simple function

$$
F_{x}=\sum_{\pi} \frac{F\left(E_{n}\right)}{\mu\left(E_{n}\right)} \chi_{E_{n}}, \quad(0 / 0)=0,
$$

where $\chi_{E_{n}}$ is the indicator function of $E_{n} \in \Sigma$. Then, as R $\phi$ nnow [7] has shown for the case $p=1$ (the same argument holds for all $p \geqq 1$ ) there exists $f \in L^{p}(\Omega, \Sigma, \mu, X)(1 \leqq p<\infty)$ such that

$$
F(E)=\int_{E} f d \mu, \quad E \in \Sigma, \text { (Bochner) }
$$

if and only if the net $\left\{F_{\pi}, \pi \in p\right\}$ is a Cauchy net in $L^{p}(\Omega, \Sigma, \mu, X)$. Now the projections $E_{\pi}$ defined on $L^{p}(\Omega, \Sigma, \mu, X)$ for each partition $\pi=\left\{E_{n}\right\}$ by

$$
E_{\boldsymbol{\pi}}(f)=\sum_{\boldsymbol{\pi}} \frac{\int_{E_{n}} f d \mu}{\mu\left(E_{n}\right)} \chi_{E_{n}}
$$


for $f \in L^{p}(\Omega, \Sigma, \mu, X)$ are contractions satisfying $E_{\pi} E_{\pi_{1}}=E_{\pi_{1}} E_{\pi}=E_{\pi_{1}}$ if $\pi \geqq \pi_{1}$. Now, evidently if $F$ is as above, then $\left\{F_{\pi}, E_{\pi}, \pi \in P\right\}$ is an abstract martingale in $L^{p}(\Omega, \Sigma, \mu, X)$, combining these facts with Theorem 1 results in the following general Radon-Nikodym theorem.

TheOREM 4. Let $(\Omega, \Sigma, \mu)$ be a finite measure space and $X$ be a Banach space. Let $F$ be a $\mu$-continuous countably additive $X$ valued set function defined on $\Sigma$. Then there exists $f \in L^{p}(\Omega, \Sigma, \mu, X)(1 \leqq p<\infty)$ such that

$$
F(E)=\int_{E} f d \mu, \quad E \in \Sigma,
$$

if and only if there exists a weakly compact set $K \subset L^{p}(\Omega, \Sigma, \mu, X)$ with the property that for each $\epsilon<0$ there exists a partition $\pi_{0}$ such that $\pi \geqq \pi_{0}$ implies $F_{\pi} \in K+\epsilon U$ where $U$ is the open unit ball of $L^{p}(\Omega, \Sigma, \mu, X)$.

\section{REFERENCES}

1. John Y. Barry, On the convergence of ordered sets of projections, Proc. Amer. Math. Soc. 5(1954), 313-314. MR 15, 964.

2. S. D. Chatterji, A note on the convergence of Banach-space valued martingales, Math. Ann. 153 (1964), 142-149. MR 28 \#4583.

3. Ralph DeMarr, A martingale convergence theorem in vector lattices, Canad. J. Math. 18 (1966), 424-432.

4. N. Dunford and J. T. Schwartz, Linear operators. I: General theory, Pure and Appl. Math., vol. 7, Interscience, New York, 1958. MR 22 \#8302.

5. J. L. Doob, Stochastic processes, Wiley, New York; Chapman \& Hall, London, 1953. MR 15, 445.

6. J. L. Kelley, General topology, Van Nostrand, Princeton, N. J., 1955. MR 16, 1136.

7. Ulf R $\phi$ nnow, On integral representation of vector valued measures, Math. Scand. 21 (1967), 45-53. MR 39 \#4354.

8. F. S. Scalora, Abstract martingale convergence theorems, Pacific J. Math. 11 (1961), 347-374. MR 23 \#A684.

UNIVERSITY OF ILLINOIS, URBANA, ILLINOIS 61801 